\title{
Revealing Antiferromagnetic Order of the Fe Monolayer on W(001): Spin-Polarized Scanning Tunneling Microscopy and First-Principles Calculations
}

\author{
A. Kubetzka, ${ }^{1, *}$ P. Ferriani, ${ }^{1}$ M. Bode,${ }^{1}$ S. Heinze,${ }^{1}$ G. Bihlmayer,${ }^{2}$ K. von Bergmann, ${ }^{1}$ \\ O. Pietzsch, ${ }^{1}$ S. Blügel, ${ }^{2}$ and R. Wiesendanger ${ }^{1}$ \\ ${ }^{1}$ Institute of Applied Physics and Microstructure Research Center, University of Hamburg, \\ Jungiusstraße 11, 20355 Hamburg, Germany \\ ${ }^{2}$ Institut für Festkörperforschung, Forschungszentrum Jülich, 52425 Jülich, Germany
}

(Received 13 August 2004; published 3 March 2005)

\begin{abstract}
We prove that the magnetic ground state of a single monolayer Fe on $\mathrm{W}(001)$ is $c(2 \times 2)$ antiferromagnetic, i.e., a checkerboard arrangement of antiparallel magnetic moments. Real space images of this magnetic structure have been obtained with spin-polarized scanning tunneling microscopy. An out-ofplane easy magnetization axis is concluded from measurements in an external magnetic field. The magnetic ground state and anisotropy axis are explained based on first-principles calculations.
\end{abstract}

The $3 d$ transition metals bcc Fe, hcp Co, and fcc Ni are known as the prototypical ferromagnets (FM). Much excitement was raised as experiments [1,2] indicated that $\mathrm{Fe}$ becomes an antiferromagnet (AFM) when stabilized in the metastable fcc phase. These experiments opened the vista that a proper control of the type of magnetic order is possible, allowing, for example, to turn a ferromagnet into an antiferromagnet or into a spin glass. However, after 40 years of research, all attempts to stabilize antiferromagnetic Fe ended up in Fe phases with fairly complex magnetic structures [3-5]. Thus, controlling the magnetic order in solids remains a challenge to solid state physics.

Low-dimensional systems offer new possibilities to tune interactions. In this Letter we propose the interface tuning of the exchange interaction as a new route to antiferromagnetism in low-dimensional systems. For one monolayer (ML) Fe on W(001) we provide a clear proof of collinear antiferromagnetic $\mathrm{Fe}$. Although this system has been studied extensively in the past, experiments could show only that the Fe ML is not ferromagnetic above $100 \mathrm{~K}$ [6,7], while theoretical predictions are still controversial $[8,9]$. Employing spin-polarized scanning tunneling microscopy (SP-STM) we obtained atomic resolution images which show that a $c(2 \times 2)$ AFM structure with an out-ofplane magnetization direction is the magnetic ground state. This is in clear contrast to the Fe ML on W(110), which is ferromagnetic with an easy axis in the film plane [10], giving evidence that the sign of the exchange interaction can be selected by the choice of the substrate orientation alone. These different experimental results are explained based on first-principles calculations.

The lack of remanence of $1 \mathrm{ML} \mathrm{Fe} / \mathrm{W}(001)$ was first discovered by spin-resolved photoemission [6] and Kerr effect measurements [7] and later interpreted on the basis of density-functional theory in the local spin-density approximation (LSDA) as an AFM ground state [8]. In these calculations the AFM state was just $10 \mathrm{meV} / \mathrm{Fe}$ atom lower than the nonmagnetic state and surprisingly the
FM state did not exist. More recent calculations based on the generalized gradient approximation (GGA), however, found a FM solution, but the AFM state was not considered [9]. Meanwhile, the experimental result, i.e., the absence of remanent magnetization, has been confirmed in numerous experiments [11-14]. To our knowledge, however, up to now no experimental technique was able to clarify whether the ground state of $1 \mathrm{ML} \mathrm{Fe} / \mathrm{W}(001)$ is paramagnetic or indeed antiferromagnetic, or even ferromagnetic with a very low Curie temperature.

Recently, it has been demonstrated that complex atomic spin structures can be resolved by SP-STM [15]. For a film with chemically equivalent atoms such as $\mathrm{Fe}$ on $\mathrm{W}(001)$, we expect the magnetic superstructure to dominate the SPSTM image [16] in the case of AFM ordering (if the magnetization direction of tip and sample are not orthogonal), while in the FM or nonmagnetic state all $\mathrm{Fe}$ atoms are equivalent.

The measurements were performed in a multichamber system with a base pressure of $p<1 \times 10^{-10} \mathrm{mbar}$, equipped with a low-temperature STM [17]. During the measurements the tip and the sample were held at $T=$ $15 \pm 1 \mathrm{~K}$. For the spin-polarized measurements we used Fe-coated W tips which are sensitive to the in-plane component of the sample magnetization in zero field. Out-ofplane sensitivity was achieved by applying a perpendicular external field of $\pm 2.5 \mathrm{~T}$. This field strength is sufficiently high to rotate the tip magnetization to out of plane [18] but leaves an antiferromagnetic sample unaffected. Fe was deposited with the clean $\mathrm{W}(001)$ substrate held at $T \approx$ $350 \mathrm{~K}$. Maps of differential conductance $(d I / d U)$ were recorded simultaneously to constant current images by adding a small modulation $\left(U_{\bmod }=10 \mathrm{mV}\right)$ to the applied gap voltage and detecting the $d I / d U$ signal by means of the lock-in technique.

A $100 \times 100 \mathrm{~nm}^{2}$ region of $1.3 \mathrm{ML}$ Fe on W(001) including a step edge at the lower right can be seen in Fig. 1(a). As described previously, islands of the second 


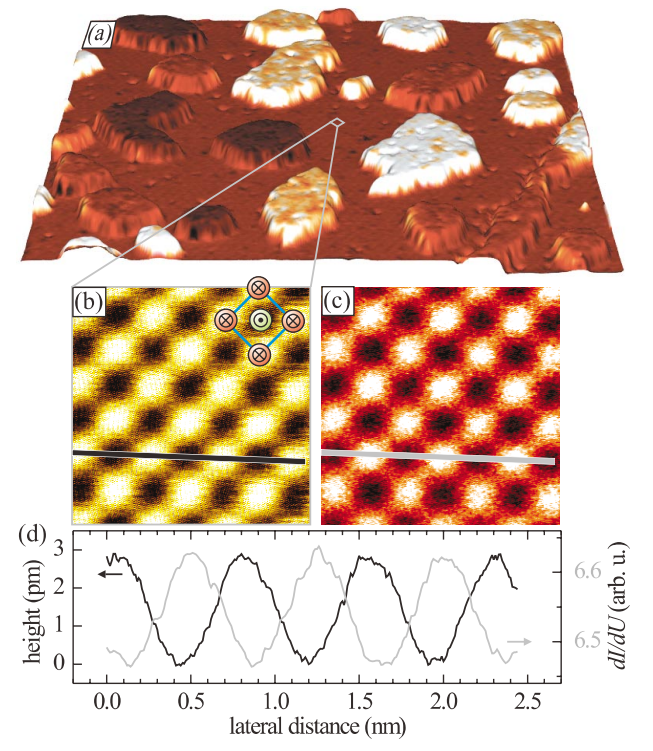

FIG. 1 (color). (a) 3D composite of topography (height) and $d I / d U$ signal (color) of a $100 \times 100 \mathrm{~nm}^{2}$ surface area of $1.3 \mathrm{ML}$ $\mathrm{Fe} / \mathrm{W}(001)(I=2 \mathrm{nA}, U=500 \mathrm{mV})$. In zero field a four stage magnetic contrast is observed on the second layer islands. In an external field of $B=+2.5 \mathrm{~T}$ the ML shows a $c(2 \times 2)$ superstructure in (b) the constant current image as well as in (c) the corresponding $d I / d U$ map $\left(2.5 \times 2.5 \mathrm{~nm}^{2}, I=3 \mathrm{nA}, U=\right.$ $-100 \mathrm{mV}$ ). (d) Line sections along the [100] direction.

layer display a four stage magnetic contrast in zero field, corresponding to the four degenerate in-plane directions [19]. Here we focus on the Fe ML. The atomic $p(1 \times 1)$ lattice of the Fe ML can be resolved with a nonmagnetic W tip (not shown), while with an Fe-coated tip in sufficiently large perpendicular fields a $c(2 \times 2)$ magnetic superstructure is observed: Fig. 1(b) displays the constant current image and Fig. 1(c) the corresponding $d I / d U$ map of a $2.5 \times 2.5 \mathrm{~nm}^{2}$ ML region at $B=+2.5 \mathrm{~T}$. The magnetic unit cell is sketched in Fig. 1(b). The line sections in Fig. 1(d) show that the $d I / d U$ map displays an inverted signal with respect to the topography [20], with a small lateral shift arising from the finite integration time of the lock-in amplifier. To unambiguously prove that the magnetic ground state is a $c(2 \times 2)$ AFM structure with an outof-plane magnetic easy axis, we have imaged a surface area twice with opposite perpendicular tip magnetizations: Figs. 2(a) and 2(b) display the same $4 \times 4 \mathrm{~nm}^{2}$ Fe ML region in an external field of $+2.5 \mathrm{~T}$ and $-2.5 \mathrm{~T}$, respectively. The native adsorbate in the center is used as a position marker. The contrast reversal which arises from switching the tip magnetization confirms the out-of-plane easy axis and the magnetic origin of the $c(2 \times 2)$ pattern. The reversal is most obvious in the corresponding line sections in Fig. 2(c). Magnetic and nonmagnetic contributions can be separated by calculating the difference and the sum of these $d I / d U$ maps, which are shown in Fig. 2(d) and 2(e), respectively. Interestingly, the "magnetic checkerboard" is visible in Fig. 2(d) even at the position of the adsorbate [21].

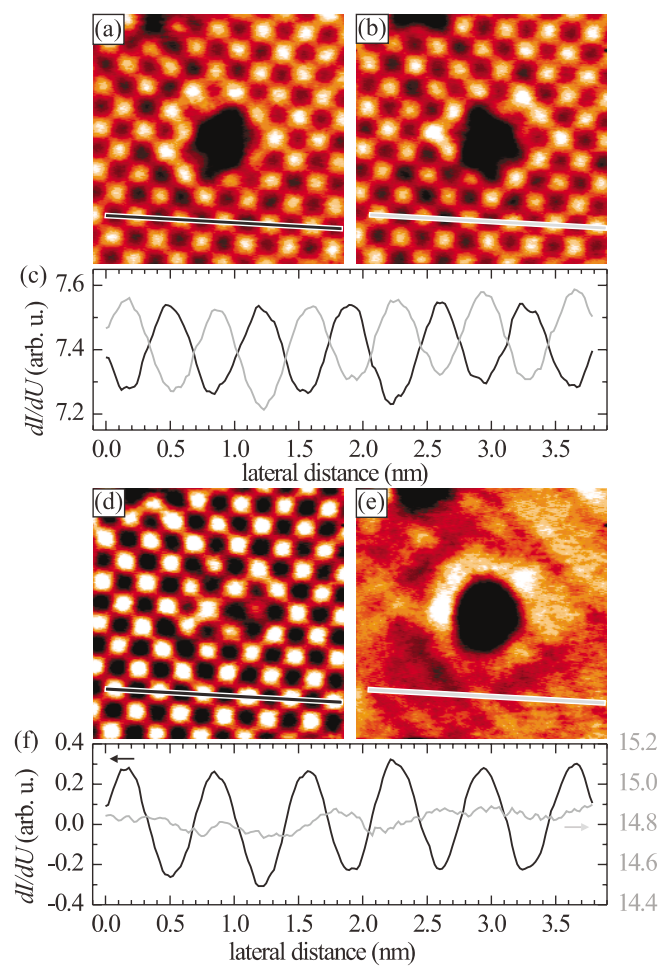

FIG. 2 (color). $\quad d I / d U$ maps of a $4 \times 4 \mathrm{~nm}^{2}$ ML region in an external field of (a) $+2.5 \mathrm{~T}$ and (b) $-2.5 \mathrm{~T}$ with a native adsorbate used as a position marker $(I=30 \mathrm{nA}, U=-40 \mathrm{mV})$. Magnetic and nonmagnetic contributions can be separated by calculating (d) the difference and (e) the sum of the images above.

To understand these experimental results and solve the theoretical inconsistencies, we performed first-principles calculations using the full-potential linearized augmented plane wave (FLAPW) method in film geometry as implemented in the FLEUR code [22]. The pseudomorphic Fe monolayer on $\mathrm{W}(001)$ was modeled by a symmetric nine layer slab with the experimental $\mathrm{W}$ lattice constant of $3.165 \AA$ and an additional Fe layer on each side of the film. We have applied the GGA of Perdew, Burke, and Ernzerhof [23]. The W $5 p$ semicore states have been described by local $p$ orbitals added to the LAPW basis set. We have used about 120 basis functions per atom, 100 $\mathbf{k}_{\|}$points in the two-dimensional Brillouin zone (2D-BZ) and muffin-tin (MT) spheres with radii of $R_{\mathrm{MT}}=2.25$ a.u. $(1$ a.u. $=0.529 \AA)$. The magnetocrystalline anisotropy energy (MAE), defined as the energy difference between the two magnetization directions, in the film plane and perpendicular to it, has been evaluated including spin-orbit coupling in a second variational procedure, using $1936 \mathbf{k}_{\|}$ points in the 2D-BZ.

In order to find the magnetic ground state we have calculated the total energy of the system as a function of the interlayer distance $d$ between the Fe monolayer and the $\mathrm{W}$ surface for the FM and $c(2 \times 2) \mathrm{AFM}$ configurations [see Fig. 3(a)] [24]. If the Fe monolayer is more than 6 a.u. from the $\mathrm{W}$ surface (not shown), the interaction is small and the monolayer can be considered in good approxima- 
tion as unsupported. In this case the FM solution has the lowest total energy. As the Fe-W interlayer distance decreases, the energy difference to the AFM solution becomes smaller until the solutions are degenerate at $d=$ 4.5 a.u. At lower interlayer distances the Fe-W hybridization increases and the $c(2 \times 2)$ AFM solution is the ground state. At the equilibrium interlayer distances of 2.44 a.u. and 2.58 a.u. for the FM and $c(2 \times 2)$ AFM states, respectively, there is a large energy difference of $160 \mathrm{meV} / \mathrm{Fe}$ atom. At even lower Fe-W interlayer distances the Fe-W hybridization quenches the magnetic moments and both solutions converge to the nonmagnetic state. Consequently, the interlayer relaxation has a strong influence on the magnitude of the magnetic moment as shown in Fig. 3(b). The moments for the relaxed FM and $c(2 \times 2)$ AFM state are $2.06 \mu_{\mathrm{B}}$ and $2.67 \mu_{\mathrm{B}}$, respectively. Our values for the Fe-W interlayer relaxation, the magnetic moments, and the energy gain for the AFM ground state are larger than those obtained in a previous calculation [8] due to the use of GGA. We have checked by a separate calculation for the FM solution that smaller magnetic moments and equilibrium interlayer distances are obtained with LSDA [see Fig. 3(b)]. Correspondingly, there is no FM solution in LSDA, while it exists in GGA in agreement with Ref. [9]. Although the W lattice constant is overestimated in GGA by $0.8 \%$, we believe that GGA is more suitable to describe this system since the LSDA systematically underestimates the interlayer distance for $3 d$ transition metal films [25] and fails to predict the correct magnetic ground state of bulk Fe, which GGA describes correctly [26].

The correlation between Fe-W hybridization and magnetic ground state can be understood based on a calculation

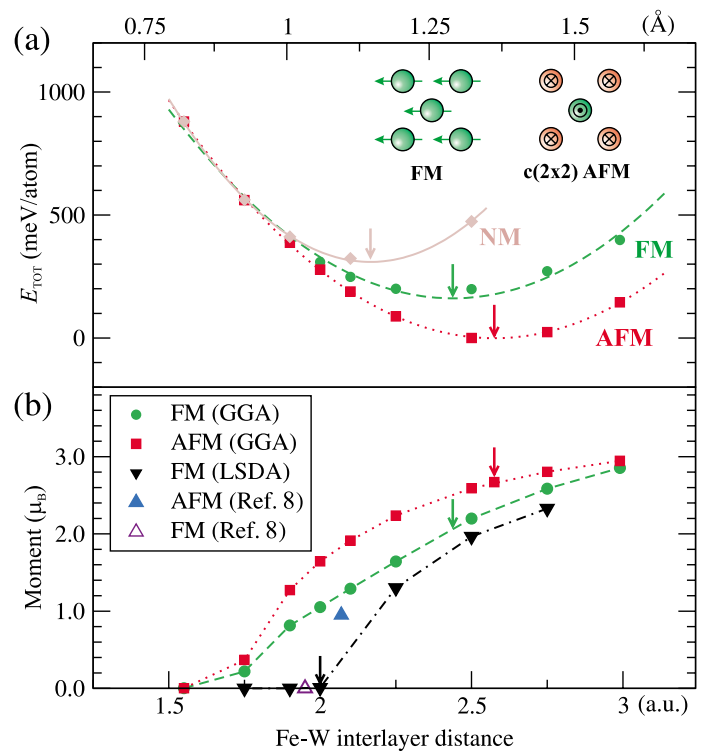

FIG. 3 (color). (a) Total energy and (b) magnetic moment of $1 \mathrm{ML} \mathrm{Fe}$ on W(001) as a function of the Fe-W interlayer distance for the two different magnetic configurations shown in the inset of (a) and for the nonmagnetic (NM) case. In (b) all triangles represent solutions obtained in LSDA. of the nonmagnetic local density of states (LDOS) as a function of interlayer distance $d$. At $d=6.0$ a.u., Fig. 4(a), there is almost no hybridization with the $\mathrm{W}$ surface, and the LDOS of Fe resembles that of an unsupported ML (UML). In accordance with the Stoner criterion, the large peak of the LDOS at the Fermi energy $E_{\mathrm{F}}$ favors ferromagnetism. At $d=4.50$ a.u., Fig. 4(b), the LDOS has broadened due to the onset of $\mathrm{Fe}-\mathrm{W}$ hybridization, and the total energies of the FM and AFM solutions become degenerate. For $d<$ 4.50 a.u. hybridization dominates the LDOS of Fe near the Fermi energy. At the AFM equilibrium interlayer distance, Fig. 4(c), we observe a splitting of the LDOS into three peaks which are also present at the $\mathrm{W}$ interface demonstrating the hybridization. Because of the reduced number of states at $E_{\mathrm{F}}$ the FM configuration becomes less favorable at the small interlayer distance. In fact, the nonmagnetic LDOS displayed in Fig. 4(c) is similar to that of the $\mathrm{Mn}$ and the $\mathrm{Cr}$ monolayer on (001) surfaces of noble metals which are prototypes for two-dimensional antiferromagnetism [27].

By including spin-orbit coupling, we have calculated the MAE. In agreement with the experiment we obtain an outof-plane easy axis with a MAE of $2.4 \mathrm{meV} / \mathrm{Fe}$ atom for the AFM ground state. For the hypothetical FM solution, however, the easy axis is in plane with a MAE of $-0.7 \mathrm{meV} / \mathrm{Fe}$ atom. Since for an Fe ML without W substrate (UML) both AFM and FM solutions possess an outof-plane easy axis with MAE of 3.2 and $1.0 \mathrm{meV} / \mathrm{Fe}$ atom, respectively, we conclude that the $\mathrm{W}$ substrate plays a key role. Nie et al. have recently shown that the $\mathrm{W}$ contribution

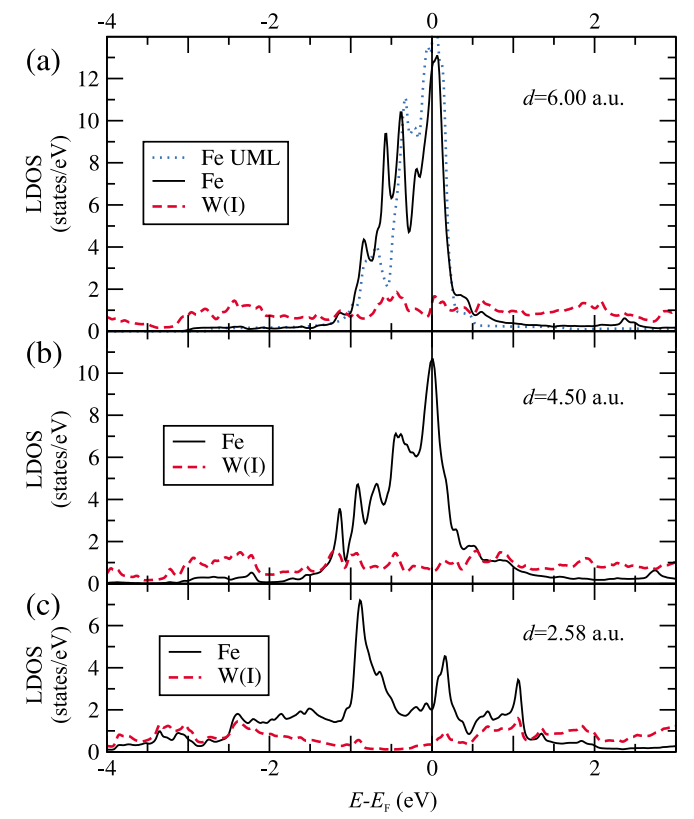

FIG. 4 (color). Nonmagnetic LDOS projected onto the Fe (solid line) and $\mathrm{W}$ (dashed line) atom at the interface of $1 \mathrm{ML}$ Fe on W(001), calculated for three different Fe-W interlayer distances $d$. In (a) the LDOS of the unsupported ML (UML) is shown for comparison (dotted line). 


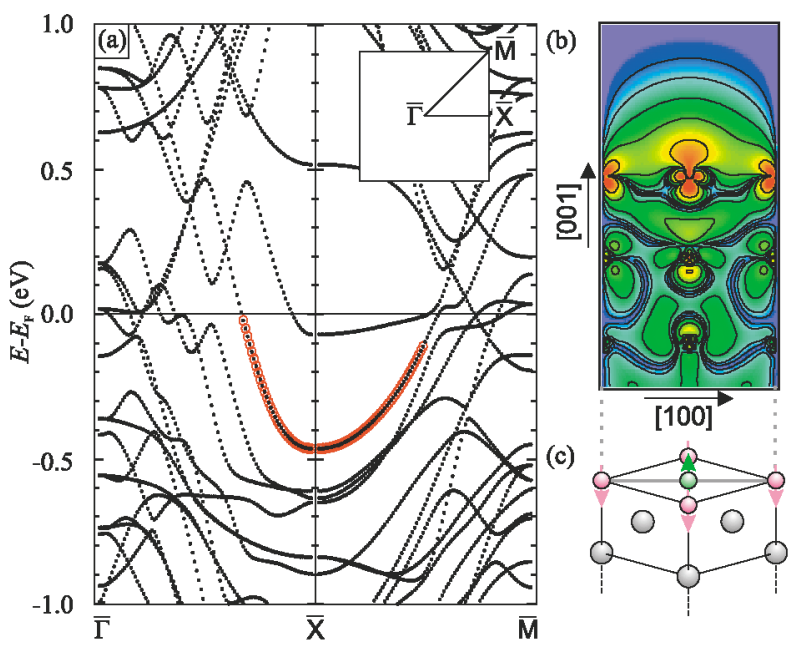

FIG. 5 (color). (a) Band structure of the $c(2 \times 2)$ AFM state of $1 \mathrm{ML} \mathrm{Fe}$ on W(001). The marked states are mainly localized in the Fe layer and produce a strong corrugation. (b) Cross section of the charge density of the majority spin channel (with respect to the magnetization of the central Fe atom) for the marked state at $\bar{X}$. (c) Top part of the film unit cell.

to the MAE forces the easy axis in plane for $1 \mathrm{ML} F e$ on $\mathrm{W}(110)$ [28]. On W(001) the situation is similar for the FM solution. For the AFM state, however, due to symmetry the $\mathrm{W}$ atoms at the interface are not spin polarized, and their direct contribution to the MAE vanishes. Thus, the magnetization axis remains out of plane as for the UML, with a smaller MAE because of $\mathrm{Fe}-\mathrm{W}$ hybridization.

Our calculations further reveal that the states responsible for the magnetic contrast observed at small bias voltages belong to a band with a minimum at $E=E_{\mathrm{F}}-0.45 \mathrm{eV}$ at the $\bar{X}$ point of the $\mathrm{BZ}$ which stretches to the Fermi energy [see Fig. 5(a)]. These states are mainly located in the Fe film and possess $d_{z^{2}}$ and $d_{x z, y z}$ character at the Fe atoms with antiparallel magnetic moments. Therefore, maxima and nodes of the wave function are alternately probed in a scan along the [100] direction and a measurable corrugation amplitude is obtained [cf. Fig. 5(b)]. Since the $d_{z^{2}}$ character corresponds to the local majority spin channel, we expect protrusions for $\mathrm{Fe}$ atoms with magnetic moment antiparallel to the tip magnetization and depressions for the parallel case, provided that the tip LDOS is dominated by minority spins.

The two-dimensional antiferromagnetic ground state of $\mathrm{Fe}$ on $\mathrm{W}(001)$ results from strong hybridization at the interface due to four nearest W neighbors. On the W(110) surface, in contrast, there are only two nearest W neighbors and the Fe monolayer is ferromagnetic. If we consider the (001) surface of Mo which is isoelectronic to W we also obtain the $c(2 \times 2) \mathrm{AFM}$ ground state for the Fe ML. In contrast, calculations for $1 \mathrm{ML} \mathrm{Fe}$ on $\mathrm{Ta}(001)$, the neighboring element of $\mathrm{W}$ in the periodic table, predict a ferromagnetic ground state. Thus, depending on the choice of the band filling, tunable by preparing proper $\mathrm{Ta}-\mathrm{W}(001)$ alloy substrates, or depending on the surface orientation, we are able to tune the competition between ferromagnetic and antiferromagnetic interactions and thus stabilize a variety of new magnetic structures which are so far unexplored.

Financial support from the DFG (Grant No. Wi 1277/ 19), the Graduiertenkolleg "Physik nanostrukturierter Festkörper," the EU project ASPRINT, the "Stifterverband für die Deutsche Wissenschaft," and the Interdisciplinary Nanoscience Center Hamburg is gratefully acknowledged.

*Corresponding author.

Electronic address: kubetzka@physnet.uni-hamburg.de

[1] S. C. Abrahams, L. Guttman, and J. S. Kasper, Phys. Rev. 127, 2052 (1962).

[2] U. Gonser et al., J. Appl. Phys. 34, 2373 (1963).

[3] Y. Tsunoda, J. Phys. Condens. Matter 1, 10427 (1989).

[4] D. Li et al., Phys. Rev. Lett. 72, 3112 (1994).

[5] D. Qian et al., Phys. Rev. Lett. 87, 227204 (2001).

[6] G. A. Mulhollan, R. L. Fink, J. L. Erskine, and G. K. Walters, Phys. Rev. B 43, 13645 (1991).

[7] J. Chen and J. L. Erskine, Phys. Rev. Lett. 68, 1212 (1992).

[8] R. Wu and A. J. Freeman, Phys. Rev. B 45, R7532 (1992).

[9] X. Qian and W. Hübner, Phys. Rev. B 67, 184414 (2003).

[10] H. J. Elmers and U. Gradmann, Appl. Phys. A 51, 255 (1990).

[11] H. J. Elmers and J. Hauschild, Surf. Sci. 320, 134 (1994).

[12] H. J. Choi et al., J. Appl. Phys. 85, 4958 (1999).

[13] W. Wulfhekel et al., Europhys. Lett. 49, 651 (2000).

[14] W. Wulfhekel et al., Phys. Rev. B 68, 144416 (2003).

[15] S. Heinze et al., Science 288, 1805 (2000).

[16] D. Wortmann et al., Phys. Rev. Lett. 86, 4132 (2001).

[17] O. Pietzsch et al., Rev. Sci. Instrum. 71, 424 (2000).

[18] A. Kubetzka et al., Phys. Rev. B 67, 020401(R) (2003).

[19] K. von Bergmann, M. Bode, and R. Wiesendanger, Phys. Rev. B 70, 174455 (2004).

[20] A. Kubetzka et al., Appl. Phys. A 76, 873 (2003).

[21] This observation indicates that the Fe AFM state is largely unaffected by the adsorbate. Since its chemical nature is unknown, though, we cannot give a detailed interpretation of the data.

[22] http://www.flapw.de.

[23] Y. Zhang and W. Yang, Phys. Rev. Lett. 80, 890 (1998).

[24] We have also studied the $p(2 \times 1)$ AFM configuration, where the Fe moments show a row-wise arrangement. The total energy curve lies between the $c(2 \times 2)$ AFM and the FM solution, with a minimum at $d=2.53$ a.u., $61 \mathrm{meV} / \mathrm{Fe}$ atom above the $c(2 \times 2) \mathrm{AFM}$ state.

[25] M. Bode et al., Phys. Rev. B 66, 014425 (2002).

[26] D. J. Singh, W. E. Pickett, and H. Krakauer, Phys. Rev. B 43, 11628 (1991).

[27] S. Blügel, D. Pescia, and P. H. Dederichs, Phys. Rev. B 39, R1392 (1989).

[28] X. Nie, G. Bihlmayer, and S. Blügel (private communication). 